\title{
Orbitofrontal Cortex Lesions Alter Anxiety-Related Activity in the Primate Bed Nucleus of Stria Terminalis
}

\author{
Andrew S. Fox, ${ }^{1,3}$ Steven E. Shelton, ${ }^{2}$ Terrence R. Oakes, ${ }^{3,4}$ Alexander K. Converse, ${ }^{3}$ Richard J. Davidson, ${ }^{1,2,3}$ \\ and Ned H. Kalin $1,2,3$ \\ Departments of ${ }^{1}$ Psychology and ${ }^{2}$ Psychiatry and the ${ }^{3}$ Waisman Laboratory for Brain Imaging and Behavior at the University of Wisconsin-Madison, \\ Madison, Wisconsin 53719, and ${ }^{4}$ Uniformed Services University of the Health Sciences, Bethesda, Maryland 20814
}

In children, behavioral inhibition (BI) in response to potential threat predicts the development of anxiety and affective disorders, and primate lesion studies suggest involvement of the orbitofrontal cortex (OFC) in mediating BI. Lesion studies are essential for establishing causality in brain-behavior relationships, but should be interpreted cautiously because the impact of a discrete lesion on a complex neural circuit extends beyond the lesion location. Complementary functional imaging methods assessing how lesions influence other parts of the circuit can aid in precisely understanding how lesions affect behavior. Using this combination of approaches in monkeys, we found that OFC lesions concomitantly alter BI and metabolism in the bed nucleus of stria terminalis (BNST) region and that individual differences in BNST activity predict BI. Thus it appears that an important function of the OFC in response to threat is to modulate the BNST, which may more directly influence the expression of BI.

\section{Introduction}

In human children, laboratory-based measures of behavioral inhibition (BI) in response to unfamiliar individuals and/or novelty are markers of anxious temperament and are early predictors of the subsequent development of anxiety and affective disorders (N. A. Fox et al., 2005). Because mechanistic studies cannot be performed in children, we developed and validated an animal model of trait-like BI in young rhesus monkeys (Kalin and Shelton, 2003). In a previous study, we demonstrated that orbitofrontal cortex (OFC) lesions decreased the expression of BI (Kalin et al., 2007). To understand the specific role the OFC plays in the neural circuit that underlies BI, we assessed regional brain metabolic activity in freely behaving OFClesioned animals and cage-mate controls using high-resolution positron emission tomography (PET).

The OFC is part of the neural circuit involved with emotion processing and appears critical for guiding and maintaining emotional responses (Milad and Rauch, 2007; Wallis, 2007). Recent work has shown OFC neurons to encode and store the relative value of different choices, and to be required for reversing the value of a cue (Wallis, 2007). Additionally, the OFC is bidirectionally connected with the subcortical components of the circuit that mediates fear and anxiety, and is thought to play an important role in the pathophysiology of anxiety and affective disorders

Received Nov. 12, 2009; revised Feb. 22, 2010; accepted April 5, 2010.

This work was supported by National Institutes of Health (NIH) Grants MH46729 and MH69315, The HealthEmotions Research Institute, Meriter Hospital, and NIH Training Grant T32-MH018931 to A.S.F. We are grateful to J. Oler, H. Van Valkenberg, T. Johnson, J. King, S. Mansavage, J. Droster, L. Greischar, A. Jahn, T. Johnstone, A. Shackman, and the staff at the Harlow Center for Biological Psychology and the National Primate Research Center at the University of Wisconsin for their technical support and Drs. Elisabeth Murray and Jocelyne Bachevalier for their neurosurgical advice.

Correspondence should be addressed to Dr. Ned Kalin, UW Psychiatric Institute and Clinics, University of Wisconsin-Madison, 6001 Research Park Boulevard, Madison, WI 53719. E-mail: nkalin@facstaff.wisc.edu.

DOI:10.1523/JNEUROSCI.5952-09.2010

Copyright $\odot 2010$ the authors $\quad 0270-6474 / 10 / 307023-05 \$ 15.00 / 0$
(Kalin and Shelton, 2003; Milad and Rauch, 2007). Studies in both OFC- and amygdala-lesioned monkeys exposed to the noeye-contact condition (NEC) of the human intruder paradigm have demonstrated replicable reductions in threat-induced freezing, which is a measure of BI (Kalin et al., 2004, 2007; Murray and Izquierdo, 2007; Machado and Bachevalier, 2008) (but see Izquierdo et al., 2005). The results of functional imaging studies in monkeys in relation to BI have not been entirely consistent with the results from the lesion studies. For example, [F-18]fluorodeoxyglucose (FDG) PET imaging in intact animals failed to find a significant correlation between OFC metabolism and either NEC-associated freezing behavior or anxious temperament, a composite measure such that individuals with higher levels of anxious temperament have increased freezing behavior, decreased vocalizations, and increased cortisol (Kalin et al., 2005; A. S. Fox et al., 2008). Instead, these functional imaging studies highlighted subcortical stress circuits, in which the amygdala and a region encompassing the bed nucleus of stria terminalis (BNST; a part of the extended amygdala) predicted individual differences in the duration of monkeys' threat-associated freezing behavior and anxious temperament (Kalin et al., 2005; A. S. Fox et al., 2008). These data suggest that the influences of the OFC on anxiety-related behavior may be indirect, as OFC lesions alter freezing, but in intact animals, brain activity in this region does not predict individual differences in freezing. The imaging data suggest that the BNST and amygdala are the proximate regions that mediate freezing. Based on these data, we hypothesized that lesions of the OFC alter freezing and BI by modulating the function of the amygdala and BNST, regions that more directly mediate anxiety and fear.

\section{Materials and Methods}

Subjects. Six male rhesus monkeys (Macaca mulatta) that received bilateral $>60 \%$ OFC aspiration lesions (which included areas $11,12,13$, and 
14) discussed in Kalin et al. (2007) and six unoperated cage-mate controls were the focus of this study. Further characterizations of lesion extent based on Walker areas are not presented due to concerns about the reliability of using MRIs after lesion to specifically quantitate damage to subregions of OFC. Each animal was injected with FDG, exposed to the NEC condition, anesthetized, and PET scanned at both time 1, where all animals were unoperated, and at time 2, which was after lesion for the experimental group (Kalin et al., 2007). Of the six pairs of animals, one pair was excluded because we were unable to obtain quality FDG-PET data for the OFC-lesioned animal at time 1. The 10 animals included in the analysis (age: mean $=2.86$ years) were matched for age, and were housed as pairs with each experimental animal living with a control animal. All testing was done in accordance with institutional guidelines.

Positron emission tomography and behavioral measurements. The six lesioned animals and their age-matched cage-mate controls were examined twice with FDG-PET (time 1, before lesion for experimental subjects and time 2, after lesion for experimental subjects) during exposure to the NEC condition of the human intruder paradigm. Individual animals were injected with FDG intravenously and placed in a test cage, where they were exposed to a modified version of the NEC condition of the human intruder paradigm. In the modified human intruder paradigm, a human experimenter entered the room and stood with their profile to the monkey, making no eye contact with the animal $2.5 \mathrm{~m}$ from the test cage. To prevent habituation during the $30 \mathrm{~min}$ of FDG uptake, the human remained in the test room for $10 \mathrm{~min}$, left the test room for $5 \mathrm{~min}$, returned for $5 \mathrm{~min}$, left for $5 \mathrm{~min}$, and returned for the remaining $5 \mathrm{~min}$. Behavior was recorded on closed circuit video, scored by trained raters blind to condition, and aggregated across the three periods of the NEC condition. Freezing was defined as a period of at least $3 \mathrm{~s}$ characterized by tense body posture without vocalizations and movement other than slow movements of the head. Cooing was defined as a vocalization made by rounding and pursing the lips with an increase then decrease in frequency and intensity. Locomotion was coded as of one or more full steps at any speed. The freezing results presented here are a subset of the results presented in Kalin et al. (2007). After the 30 min of FDG uptake, animals were anesthetized with ketamine $(15 \mathrm{mg} / \mathrm{kg})$ and were administered intramuscular atropine sulfate $(0.27 \mathrm{mg})$. They were then fitted with an endotracheal tube, to administer $1-2 \%$ isoflurane gas anesthesia. The animal was then placed in the microPET P4 scanner (Concorde Microsystems) (Tai et al., 2001). Sixty-minute emission PET scans were reconstructed using filtered back projection and reflect the integrated brain metabolism that occurred during the $\sim 30$ min of FDG uptake.

Preprocessing. Time-2 (postsurgical for the experimental group) PET scans were masked to exclude prefrontal regions where anatomy may have changed in the experimental group, and posterior brain regions that were misplaced outside of the PET scanner field of view in some of the presurgery images [see Kalin et al. (2005) for additional information]. Each animal's time-2 masked images were transformed using a sixparameter rigid-body transformation to match their prelesion PET scan. Transformations were applied to the unmasked time-2 PET scans and manually inspected by A.S.F. to ensure that each subject's time-2 PET scans was accurately aligned to the time-1 PET image. Nonlinear transformations based on the time-1 images (computed using AIR5) were applied to the time- 2 images, resulting in time-2 images in the standard space of Paxinos, Huang, and Toga (Woods et al., 1998; Paxinos et al.,
1999). Additional details on this registration can be found in Kalin et al. (2005). To facilitate interscan comparisons, images were globally scaled by adjusting the mean, based on a partial brain ROI created by intersecting the masked images used for alignment (A. S. Fox et al., 2005; Kalin et al., 2005). Each globally scaled image was smoothed using a $4 \mathrm{~mm}^{3}$ full-width at half-maximum (FWHM) Gaussian kernel to account for slight across-subject variation in anatomy and to enforce a normal distribution.

Statistical analyses. Statistical analyses were performed using standard voxelwise regression techniques. To verify lesion efficacy in decreasing OFC metabolism, a region of interest based on the target lesion area was drawn on an MRI template, and relative glucose metabolism within this region was extracted for each subject. This effect was highly significant, suggesting our analysis was sensitive to the effects of the lesion (data not shown). To assess brain differences in non-OFC regions, analyses were performed using a voxelwise search within regions where data were accurately aligned to standard space. Posterior visual regions were excluded from the analyses, since this region was outside of the scanner field of view in some of the prelesion PET scans (see Kalin et al., 2005), and anterior regions (anterior to the genu of the corpus callosum) were excluded because we could not accurately account for lesion-induced deformations of brain structure within these regions. Within trusted regions, we identified regions $>8 \mathrm{~mm}^{3}$ (the FWHM resolution of the scanner) that showed a significant lesion/no lesion by time-1/time-2 interaction $(p<0.05$, two-tailed uncorrected) and a significant time-2 main effect of group (lesion vs no lesion) ( $p<0.005$, two-tailed uncorrected). Follow-up analyses were performed in SPSS, focusing on linear regressions on the mean values from the clusters identified in the voxelwise analysis. To test the effects of laterality, we also extracted mean values from the opposing hemisphere of significant clusters, and performed a lesion/no lesion by time 1/time 2 by left-hemisphere/righthemisphere interaction. 


\begin{tabular}{|c|c|c|c|c|c|c|c|c|}
\hline \multirow[b]{2}{*}{$+1-$} & \multirow[b]{2}{*}{ Cluster region } & \multirow[b]{2}{*}{ Volume (in $\mathrm{mm}^{3}$ ) } & \multirow[b]{2}{*}{ Cluster hemisphere } & \multirow[b]{2}{*}{ Peak location region } & \multirow{2}{*}{$\begin{array}{l}\text { Peak } t \text { value } \\
\text { (postlesion) }\end{array}$} & \multicolumn{3}{|c|}{$\begin{array}{l}\text { Peak location relative to anterior } \\
\text { commissure (in } \mathrm{mm} \text { ) }\end{array}$} \\
\hline & & & & & & $x$ & $y$ & $z$ \\
\hline+ & Entorhinal cortex & 28 & $\mathrm{~L}$ & Rostral entorhinal cortex & 15.32 & -7.5 & -0.625 & -14.375 \\
\hline+ & Postcentral gyrus & 9 & $\mathrm{R}$ & Area 3 & 14.44 & 25 & 1.875 & 4.375 \\
\hline+ & Bed nucleus of stria terminalis region & 8 & $\mathrm{~L}$ & Bed nucleus of stria terminalis & 7.17 & -4.375 & 1.25 & -0.625 \\
\hline- & Parietal & 81 & $\mathrm{R}$ & Parietal area PG & 16.75 & 13.125 & -23.75 & 18.125 \\
\hline- & $\mathrm{ACC}$ & 43 & Midline & Areas $6 / 32$ and $23 c$ & 13.69 & -0.625 & -5.625 & 15.625 \\
\hline- & Motor & 12 & $\mathrm{R}$ & Area 4 & 29.28 & 6.875 & -3.125 & 21.25 \\
\hline- & White matter near OFC & 11 & $\mathrm{R}$ & & 10.52 & 11.875 & 9.375 & 5.625 \\
\hline- & Motor & 10 & $\mathrm{R}$ & Area 6 & 8.74 & 13.75 & -0.625 & 18.75 \\
\hline
\end{tabular}

Regions where we observed a significant interaction ( $p<0.05$, two-tailed uncorrected) and where control animals showed greater metabolism than 0FC-lesioned animals at time 2 (after lesion; $p<0.005$, two-tailed uncorrected). Regions are presented with the direction of the correlation, brain regions involved, volume, and hemisphere of cluster. We also report the local maxima for each statistical cluster with its corresponding $t$ value and location (in millimeters relative to the anterior commissure). Note that our analyses did not include areas $>20 \mathrm{~mm}$ posterior to the anterior commissure or areas anterior to the genu of the corpus callosum (see Materials and Methods for details).

\section{Results}

We first examined the effects of OFC lesions on freezing behavior that occurred during the period of FDG uptake. Consistent with Kalin et al. (2007), we observed that OFC-lesioned animals, compared to intact animals, exhibited significantly less freezing when tested during the time-2 FDG-uptake period $\left(t_{(8)}=2.371, p=\right.$ 0.026 ) (see supplemental note, available at www.jneurosci.org as supplemental material; Fig. 1b) (Kalin et al., 2007). Further analyses revealed a significant increase in freezing duration within the control group $\left(t_{(4)}=4.848, p=0.008\right)$, with no group differences at time 1 or any significant decrease in freezing behavior in the experimental group $(p>0.5)$ (additional discussion can be found in supplemental material, available at www.jneurosci.org).

Based on the hypothesis that the OFC indirectly influences freezing by modulating amygdala and BNST activity, we predicted that OFC lesions would decrease activity in the amygdala and BNST. Results of the voxelwise analyses demonstrated that NEC-exposed OFC-lesioned animals had significantly $(p<$ 0.005 , two-tailed uncorrected time- 2 effect and a $p<0.05$, twotailed uncorrected interaction) lower glucose metabolism in a region that overlapped with the BNST (Fig. 1b) and additionally in the entorhinal cortex. The analysis also revealed increased activation in OFC-lesioned animals in regions of parietal cortex (LIP), midcingulate gyrus, and motor cortex (for a complete list of affected regions, see Table 1). Analyses of asymmetry revealed no group by time by hemisphere interaction in BNST metabolism $\left(F_{(4,25)}=1.44, p=0.25\right)$, suggesting no significant differences in BNST laterality. Although we did not observe significant effects of the OFC lesions on amygdala metabolism, this null finding may result from a lack of statistical power as previously published associations between freezing behavior and amygdala metabolism suggest a relatively low zero-order correlation that gets stronger when combined with other measures not discussed in this manuscript (Kalin et al., 2005).

To further examine the role of the BNST region in BI, we investigated the correlation between brain activity in the BNST region with freezing behavior at time 2 . Because we hypothesized that OFC lesions were mediated by the BNST, and would not alter the relationship between BNST and freezing, we predicted that these data would replicate the previously observed positive correlation between BNST and freezing behavior (Kalin et al., 2005). Our findings demonstrated that mean metabolic activity in the BNST region that showed a group difference between lesioned and nonlesioned animals strongly predicted freezing (Spearman's $\rho=0.782, p=0.008$; controlling for age in both variables) (Fig. 2). To determine the specificity of this correlation to freezing behavior, we performed the same analyses correlating indi-

\section{Bed Nucleus of Stria Terminals (BNST) Region Metabolism predicts Freezing}

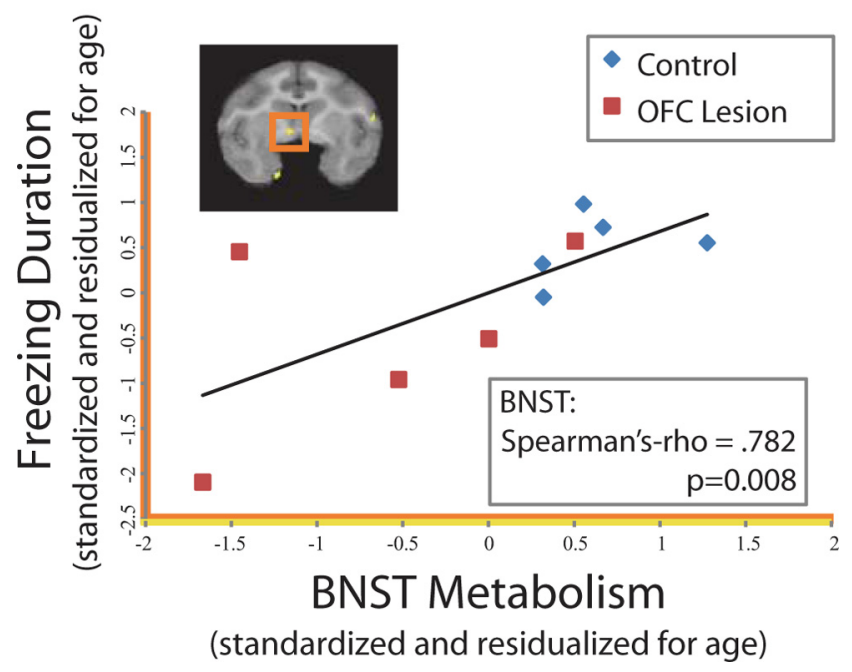

Figure 2. BNST region metabolism predicts freezing. Scatter plot demonstrating that the relationship between freezing duration (log-scaled, residualized for age, and $z$-scored) and mean FDG values at time 2 from the functionally defined BNST region (residualized for age and $z$-scored; $p<0.005$ two-tailed uncorrected, yellow in Fig. 1c and brain overlay; residualized for age and $z$-scored). These values represent a replication of the relationship between BNST region metabolism and freezing duration previously reported in Kalin et al. (2005) across the OFClesioned animals and their cage-mate controls.

vidual differences in BNST region activity with individual differences in affiliative vocalizations (coo calls) and locomotion and found that neither were significantly correlated with BNST region metabolism ( $p$ values $>0.174$ ). To ensure that the BNST region was accounting for the majority of the variance associated with freezing behavior, we entered each of the regions affected by the OFC lesions into a hierarchical linear regression with the BNST region. This analysis revealed that metabolic activity in no other region significantly accounted for additional variance in freezing behavior beyond that accounted for by the BNST region ( $F$-change values $<2.402$, $p$ values $>0.165$ ). These analyses suggest that the BNST may be the critical brain region associated with the effect of the OFC lesions on decreasing freezing behavior. Additionally, this finding is consistent with previous research suggesting that motor, parietal, and midcingulate regions may be related to specific operations associated with BI, such as alterations in locomotion and visual attention, rather than the altered motivation associated with BI itself (Paus, 2001; Gottlieb, 2007). 


\section{Discussion}

Using a combined lesion and imaging approach in an established primate model, we demonstrate an interaction between the OFC and the BNST region that sheds light on how the OFC modulates trait-like anxiety-related responses in primates. OFC lesions altered freezing, a behavior that is critical and adaptive in helping an animal remain undiscovered in the face of a potential threat. The current data demonstrate that OFC lesion-induced changes in freezing could be accounted for by alterations in BNST activity.

Recent research has highlighted a number of important functions of the OFC that help elucidate its role in anxiety-related behavior. In particular, electrophysiological recordings in monkeys and functional imaging in humans have shown this region to track the relative value of choices and maintain this representation over time (Kringelbach and Rolls, 2004; Wallis, 2007). Additionally, lesion studies have demonstrated the OFC to be critical for updating the value of reward-related cues (Wallis, 2007). The OFC may respond similarly to threat-related cues such that in the NEC paradigm the OFC could be accumulating and maintaining the emotional representation of the human intruder to facilitate adaptive behavioral responses. Importantly, this is consistent with structural and functional differentiation of the OFC into "medial" and "orbital" OFC networks, with the orbital OFC (the region lesioned in this study) demonstrating more activation to cues that signal negative outcomes (Kringelbach and Rolls, 2004; Price, 2007). Based on the data from the current study, we further suggest that the behavioral actions induced by the perception of threat cues are implemented via the OFC's influence on the BNST region.

The relation we observed between individual differences in BNST region metabolism and trait-like anxiety agrees with our previous work as well as rodent work examining the functional significance of BNST activity (Walker et al., 2003; Kalin et al., 2005; A. S. Fox et al., 2008). The BNST is part of the limbic system and basal forebrain, with the posterior edge near the central nucleus of the amygdala ( $\mathrm{CeA})$ and the anterior edge bordering on the nucleus accumbens (Heimer et al., 1997). The similar development, cytoarchitecture, and connectivity of the BNST and CeA have led researchers to group these regions into the broader construct of the "extended amygdala" (Heimer, 2003). Because the extended amygdala receives substantial input from other amygdala nuclei and projects to the brainstem and hypothalamic regions required to mount a stress response, the extended amygdala is ideally situated to be the interface to the motor responses associated with emotional reactions (Heimer et al., 1997). More recently, Walker and Davis have suggested a functional dissociation within the extended amygdala, such that the CeA is necessary for rapid-onset, short-duration, acute fear responding to specific threat, and the BNST is associated with slower-onset, longerlasting responses associated with anxiety and BI (Walker et al., 2003). These data are consistent with the interpretation that OFC lesions alter metabolism in the BNST region, which in turn initiates BI measured in response to a relatively long (30 $\mathrm{min})$ exposure to the NEC condition. Importantly, our findings are also highly consistent with ongoing work in rodents demonstrating the BNST region to mediate individual differences in anxiety, and our work in monkeys suggesting that serotonin transporter availability in the BNST may represent a complementary mechanism for altering BNST activity (Duvarci et al., 2009; Oler et al., 2009).

Interestingly, anatomical tract tracing studies in primates have not observed the orbital OFC network to be directly connected to the BNST. Retrograde tracing studies measuring the direct projections to the orbital OFC have failed to label neurons in the BNST, and the anterograde studies investigating the projections from the OFC to the BNST have not been published (Price, 2007). Thus, it remains unclear whether the OFC lesions are decreasing freezing behavior via direct or indirect connections to the BNST (e.g., via known thalamic, insular, or amygdalar connections), and highlights the fact that these two regions represent only a portion of the functional network that underlies BI (Ghashghaei et al., 2007).

Although this sample size is consistent with that of other studies investigating the effects of targeted lesions on behavior, it is substantially underpowered for analyses that attempt to understand the relation between individual differences in lesion extent with brain activity and behavior. Future work with larger sample sizes will facilitate an understanding of the relationships among lesion location, remaining functional activation within the lesioned areas, and lesion-induced behavioral alterations.

In conclusion, these data suggest that the OFC does not directly alter freezing per se, but rather the behavioral effects of OFC lesions result from the removal of descending modulatory inputs (direct or indirect) to the BNST region. It follows that alterations in OFC function may underlie difficulties in the adaptive regulation of anxiety, which is thought to be a cardinal feature of anxiety and affective disorders (Kalin and Shelton, 2003). In contrast to the strategy of using either selective lesion techniques or functional imaging to understand brain-behavior relations, this study underscores the power of combining these methods to better understand the complex interactions among components of relevant neural circuits.

\section{References}

Duvarci S, Bauer EP, Paré D (2009) The bed nucleus of the stria terminalis mediates inter-individual variations in anxiety and fear. J Neurosci 29:10357-10361.

Fox AS, Oakes TR, Shelton SE, Converse AK, Davidson RJ, Kalin NH (2005) Calling for help is independently modulated by brain systems underlying goal-directed behavior and threat perception. Proc Natl Acad Sci U S A 102:4176-4179.

Fox AS, Shelton SE, Oakes TR, Davidson RJ, Kalin NH (2008) Trait-like brain activity during adolescence predicts anxious temperament in primates. PLoS ONE, 3:e2570.

Fox NA, Henderson HA, Marshall PJ, Nichols KE, Ghera MM (2005) Behavioral inhibition: linking biology and behavior within a developmental framework. Annu Rev Psychol 56:235-262.

Ghashghaei HT, Hilgetag CC, Barbas H (2007) Sequence of information processing for emotions based on the anatomic dialogue between prefrontal cortex and amygdala. Neuroimage 34:905-923.

Gottlieb J (2007) From thought to action: the parietal cortex as a bridge between perception, action, and cognition. Neuron 53:9-16.

Heimer L (2003) A new anatomical framework for neuropsychiatric disorders and drug abuse. Am J Psychiatry 160:1726-1739.

Heimer L, Harlan RE, Alheid GF, Garcia MM, de Olmos J (1997) Substantia innominata: a notion which impedes clinical-anatomical correlations in neuropsychiatric disorders. Neuroscience 76:957-1006.

Izquierdo A, Suda RK, Murray EA (2005) Comparison of the effects of bilateral orbital prefrontal cortex lesions and amygdala lesions on emotional responses in rhesus monkeys. J Neurosci 25:8534-8542.

Kalin NH, Shelton SE (2003) Nonhuman primate models to study anxiety, emotion regulation, and psychopathology. Ann NY Acad Sci 1008:189-200.

Kalin NH, Shelton SE, Davidson RJ (2004) The role of the central nucleus of the amygdala in mediating fear and anxiety in the primate. J Neurosci 24:5506-5515.

Kalin NH, Shelton SE, Fox AS, Oakes TR, Davidson RJ (2005) Brain regions associated with the expression and contextual regulation of anxiety in primates. Biol Psychiatry 58:796-804.

Kalin NH, Shelton SE, Davidson RJ (2007) Role of the primate orbitofrontal cortex in mediating anxious temperament. Biol Psychiatry 62:1134-1139. 
Kringelbach ML, Rolls ET (2004) The functional neuroanatomy of the human orbitofrontal cortex: evidence from neuroimaging and neuropsychology. Prog Neurobiol 72:341-372.

Machado CJ, Bachevalier J (2008) Behavioral and hormonal reactivity to threat: effects of selective amygdala, hippocampal or orbital frontal lesions in monkeys. Psychoneuroendocrinology 33:926-941.

Milad MR, Rauch SL (2007) The role of the orbitofrontal cortex in anxiety disorders. Ann N Y Acad Sci, 1121:546-561.

Murray EA, Izquierdo A (2007) Orbitofrontal cortex and amygdala contributions to affect and action in primates. Ann N Y Acad Sci 1121:273-296.

Oler JA, Fox AS, Shelton SE, Christian BT, Murali D, Oakes TR, Davidson RJ, Kalin NH (2009) Serotonin transporter availability in the amygdala and bed nucleus of the stria terminalis predicts anxious temperament and brain glucose metabolic activity. J Neurosci 29:9961-9966.

Paus T (2001) Primate anterior cingulate cortex: where motor control, drive and cognition interface. Nat Rev Neurosci 2:417-424.
Paxinos G, Huang X, Toga A (1999) The rhesus monkey brain in stereotaxic coordinates. San Diego: Academic.

Price JL (2007) Definition of the orbital cortex in relation to specific connections with limbic and visceral structures and other cortical regions. Ann N Y Acad Sci 1121:54-71.

Tai C, Chatziioannou A, Siegel S, Young J, Newport D, Goble RN, Nutt RE, Cherry SR (2001) Performance evaluation of the microPET P4: a PET system dedicated to animal imaging. Phys Med Biol 46:1845-1862.

Walker DL, Toufexis DJ, Davis M (2003) Role of the bed nucleus of the stria terminalis versus the amygdala in fear, stress, and anxiety. Eur J Pharmacol 463:199-216.

Wallis JD (2007) Orbitofrontal cortex and its contribution to decisionmaking. Annu Rev Neurosci 30:31-56.

Woods RP, Grafton ST, Holmes CJ, Cherry SR, Mazziotta JC (1998) Automated image registration: I. General methods and intrasubject, intramodality validation. J Comput Assist Tomogr 22:139-152. 